\title{
Incidence of deepening of upper eyelid sulcus after topical use of tafluprost ophthalmic solution in Japanese patients
}

This article was published in the following Dove Press journal:

Clinical Ophthalmology

12 July 2013

Number of times this article has been viewed

\author{
Katsuhiko Maruyama' \\ Asako Tsuchisaka' \\ Jumpei Sakamoto' \\ Shiroaki Shirato ${ }^{2}$ \\ Hiroshi Goto' \\ 'Department of Ophthalmology, \\ Tokyo Medical University, Tokyo, \\ Japan; ${ }^{2}$ Yotsuya Shirato Eye Clinic, \\ Tokyo, Japan
}

\begin{abstract}
Purpose: To investigate the incidence of deepening of upper eyelid sulcus (DUES) with topical use of tafluprost in Japanese glaucoma patients.

Methods: This study was a prospective, open-label, non-controlled case series conducted at a single center. Thirty-six primary open-angle glaucoma Japanese patients who had no history of surgery were enrolled. The patients were prescribed $0.0015 \%$ topical tafluprost once daily to one eye that had the more severe visual field disorder, and observed during outpatient visits before and at 30,60, and 90 days after starting treatment. At every visit, three examiners judged the presence of DUES unanimously by facial photographs including eyebrows and lower eyelids. The primary outcome variable was the incidence of DUES at the end of day 90 in the study as assessed by observer-masked facial photography. The patients were also asked if they noticed any subjective symptom of DUES. Gender, refraction, and intraocular pressure were evaluated as potential risk factors.
\end{abstract}

Results: Thirty-two patients completed this clinical trial. DUES were identified objectively in $19 \%(6 / 32)$ of the patients after 90 days of treatment. The incidence was not related to gender, refraction, baseline or post-treatment intraocular pressure, or intraocular pressure reduction. Finally, 17\% (1/6) of patients with objectively diagnosed DUES noticed the presence of DUES by themselves. No patient dropped out of the study because of DUES.

Conclusion: Similar to other prostaglandin analogs, topical use of tafluprost ophthalmic solution is associated with DUES as a local adverse reaction.

Keywords: prostaglandins, tafluprost, eyelid, deepening, side effect

\section{Introduction}

Prostaglandin analogs have been approved as first-line drugs for glaucoma because of their potent intraocular pressure (IOP) lowering activity and few systemic adverse effects. However, local adverse events, such as iris pigmentation, increasing eyelash growth, and conjunctival hyperemia are well recognized. ${ }^{1}$ Recently, deepening of the upper eyelid sulcus (DUES) associated with the use of bimatoprost, ${ }^{2-9}$ travoprost, $^{6,7,9-12}$ latanoprost, ${ }^{9,13}$ or tafluprost ${ }^{9}$ has been reported as a local side effect.

Tafluprost is a prostaglandin F2 alpha analog, which has an IOP lowering effect and safety comparable with latanoprost in primary open-angle glaucoma (POAG) and ocular hypertension. ${ }^{14,15}$ Although tafluprost was launched in 2008 in Japan, only one retrospective report of DUES related to the use of tafluprost was published recently. ${ }^{9}$

The purpose of this study was to prospectively investigate the incidence of DUES in patients receiving tafluprost ophthalmic solution for POAG, and analyze the
Correspondence: Katsuhiko Maruyama Department of Ophthalmology,

Tokyo Medical University,

6-7-I Nishi-shinjuku, Shinjuku-ku,

Tokyo 160-0023, Japan

Tel +8I 333426 I I I

Fax +8I 333469170

Email kacchan@tokyo-med.ac.jp 
background factors related to this complication in Japanese patients.

\section{Subjects and methods}

This was an observer-masked, open-label prospective study. Thirty-six consecutive patients with POAG who started to receive $0.0015 \%$ tafluprost (Tapros; Santen Pharmaceutical Co, Ltd, Osaka, Japan) unilaterally at Tokyo Medical University between July 2010 and December 2011 were enrolled in this study. All patients were Japanese with dark brown irises and no history of intraocular surgeries including laser treatment. Patients who had a history of extraocular surgeries such as orbital or eyelid surgery, and patients with endocrinological or neurological diseases that may affect the shape of eyelids were excluded. Those who were already on ocular hypotensive drugs discontinued their use for $>4$ weeks before entering the study. This study was conducted in accordance with the Declaration of Helsinki and the study protocol was approved by the ethical committee of Tokyo Medical University. Informed consent to participate in this study was obtained from all the patients.

Tafluprost was administered once daily between $20 \mathrm{~h} 00$ and 22:00 to the eye that had more severe visual field disorder or the eye with higher IOP in the case that the visual field disorder was the same in both eyes. Prior to the beginning of unilateral treatment with tafluprost (baseline) and 30, 60, and 90 days after starting treatment, IOP and ocular surface conditions were examined routinely by slit lamp. Facial photographs, including eyebrows and lower eyelids, were taken by two of the authors (KM and AT) at baseline and 30, 60, and 90 days after starting treatment. All photographs were taken in the vision testing room (illuminated with fluorescent lamp at an illumination level of 50 lux or above) under the same photographic conditions as far as possible. Each photograph was taken with no make-up around the eyes, while the subject was seated with their head supported by a head rest and facing forward, in a natural expression without deliberately opening the eyes widely. A 3.2 mega-pixel digital camera (IXY DIGITAL 40; Canon Inc, Tokyo, Japan) was used to take the photograph at a distance of $50 \mathrm{~cm}$ and without using the flash. Photographs that were not blurred were used for evaluation. Post-treatment IOP was obtained from IOP measurements at the three outpatient visits during treatment. IOP reduction was calculated by subtracting post-treatment IOP from baseline IOP. At each visit, patients were asked whether they noticed any local or systemic symptom. All examinations were performed between 9:00 and 11h00. The examination was performed within a window of 2 hours in individual patients over the study period.
For evaluating DUES, the photographs taken at baseline and 30, 60, and 90 days were edited using Photoshop Elements 10 (Adobe Systems Inc, Tokyo, Japan) to a size that spans the eyebrows of both eyes to the checks vertically and the temples of both sides horizontally. Then, the images obtained at baseline and after 30,60, and 90 days of treatment were presented on a 23-inch liquid crystal display (Inspiron One; Dell Inc, Kanagawa, Japan). Three examiners (KM, AT and JS) evaluated the images independently by the following procedures. Signs of DUES were defined as: (1) deepening of the eyelid fold; (2) formation of a new eyelid fold; and (3) increased hollowing of the upper eyelid. After comparing the images before and after treatment and between the administered and non-administered eye, if at least one DUES sign was observed in the administered eye but not in the non-administered eye, the case was evaluated as DUES positive. The decisions of the fellow examiners were not available to an examiner during viewing and judging. After analyzing all the evaluation results from the three examiners, a case was rated as positive when there was consensus among all three examiners. The primary outcome variable was the incidence of DUES at the end of day 90 in the study as assessed by observer-masked facial photography. At each visit, patients were asked if they noticed any sign of DUES subjectively.

All data are presented as mean \pm standard deviation and analyzed using MedCalc version 12.1.4.0 (MedCalc Software bvba, Mariakerke, Belgium). Student's $t$-test was used to compare age, refraction, baseline IOP, post-treatment IOP, and IOP reduction between the groups with and without DUES. Fisher's exact test was used to compare gender distribution.

\section{Results}

\section{Demographic data}

Among 36 subjects, one stopped visiting the hospital after 30 days, one discontinued tafluprost after 30 days because of conjunctival injection, and two changed to other prostaglandin analogs because of insufficient IOP lowering effect at 90 days. Eventually, 32 patients completed 90 days of treatment and examinations. The patient group comprised 19 men and 13 women. The demographic data of the 32 patients is shown in Table 1. The eye administered tafluprost was the right eye in 15 patients and the left eye in 17 patients.

\section{Incidence of DUES and subjective symptoms}

By evaluating the photographs, the proportion of patients rated as DUES positive was 19\% (6 of 32) by KM, 
Table I Demographic data of 32 patients

\begin{tabular}{lcl}
\hline & $\begin{array}{l}\text { Mean } \pm \text { standard } \\
\text { deviation }\end{array}$ & $\begin{array}{l}\text { Minimum- } \\
\text { maximum }\end{array}$ \\
\hline Age (years) & $52.5 \pm 14.5$ & $28-80$ \\
Refraction (diopter) & $-5.42 \pm 3.32$ & $-13.00-(+0.38)$ \\
Baseline IOP $(\mathrm{mmHg})$ & $15.7 \pm 2.9$ & $12-26$ \\
\hline
\end{tabular}

Abbreviation: IOP, intraocular pressure.

$22 \%$ ( 7 of 32) by AT, and 19\% (6 of 32) by JS. Among three examiners, the results of KM and JS were identical for all positive cases, and the results of AT differed from those of KM and JS in only one subject. From the above results, excluding one patient with inconsistent judgment, the DUES positive rate was $19 \%$. In all the DUES positive cases, all three examiners concurred on the time of onset of DUES. In the six DUES positive cases, the DUES positive rate was $0 \%$ ( 0 of 32 patients) at 30 days, $9 \%$ ( 3 of 32 patients) at 60 days, and 19\% (6 of 32 patients) at 90 days (Figure 1). One of six (17\%) patients with objectively detected DUES noticed DUES subjectively at 90 days of treatment. Figure 2 shows all the cases diagnosed with DUES. No patient discontinued using tafluprost ophthalmic solution due to subjective DUES symptom.

\section{Factors contributing to DUES}

The results of analyses of factors affecting the incidence of DUES are shown in Table 2. The incidence of DUES was not related to age, gender, refraction, baseline or post-treatment IOP, or IOP reduction.

\section{Discussion}

This is the first report of a prospective study investigating the incidence of DUES associated with topical use of tafluprost evaluated by objective examination of serial photographs.

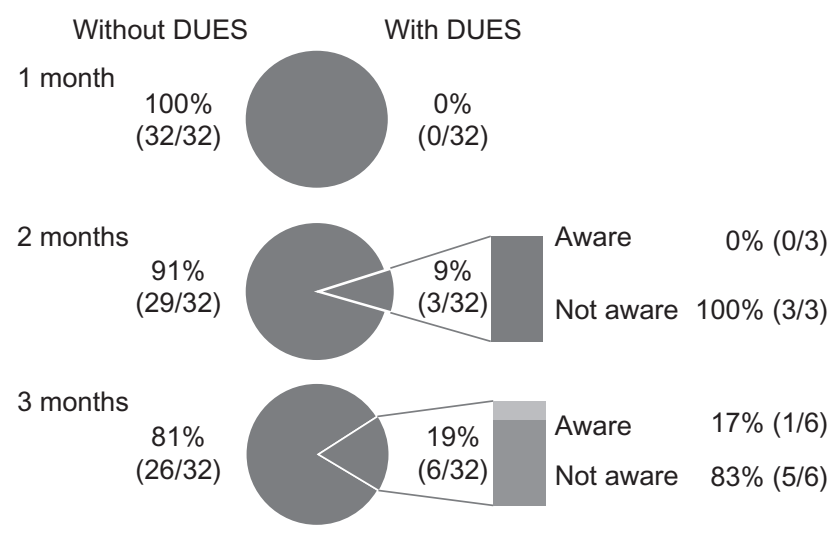

Figure I Prevalence of DUES diagnosed objectively, and the proportions of patients aware of subjective symptoms.

Abbreviation: DUES, deepening of upper eyelid sulcus.
In addition, the present study is the first to examine the incidence of DUES when a prostaglandin analog is used in unilateral eyes of glaucoma patients.

In case reports of DUES associated with prostaglandin analogs, the causative drugs were bimatoprost, ${ }^{2-7}$ travoprost, ${ }^{6,710,16}$ and latanoprost. ${ }^{13}$ In a recent report comparing the incidence of DUES caused by five prostaglandin analogs (latanoprost, travoprost, bimatoprost, tafluprost, and isopropyl unoprostone), DUES was observed also in patients treated with tafluprost (18\% assessed by photographs), ${ }^{9}$ but the study was retrospective and the true incidence remains to be confirmed. There were two prospective studies on the incidence of DUES after using topical prostaglandin analogs. Aihara et $\mathrm{al}^{8}$ studied 25 Japanese patients with open-angle glaucoma already treated with latanoprost for at least 6 months, and latanoprost was switched to bimatoprost aiming to further lower IOP. The incidence of DUES in these patients was evaluated by comparing photographs of the eyelid region taken before and after switching. The rates of DUES were $44 \%$ and $60 \%$ after 1 and 3 months of treatment, respectively, and remained unchanged at 6 months. In another study of 32 patients who had been treated unilaterally with travoprost for at least 6 months and then started to receive bilateral treatment, DUES was detected in $34 \%$ of the patients after 2 months of treatment, increasing to $53 \%$ after 4 months and remained unchanged at $53 \%$ after 6 months. ${ }^{12}$ Compared to these results, our finding suggests that the incidence of DUES caused by topical use of tafluprost is lower than that for bimatoprost and travoprost. However, given the small sample size and short follow-up period of the present study, more detailed comparative studies on the incidence of DUES associated with various prostaglandin analogs are needed.

The reason for the different incidence of DUES for various prostaglandin analogs is unknown. The development of DUES is suspected to be associated with the lipolytic action of prostaglandin analogs, and this mechanism has been proven by a magnetic resonance imaging (MRI) study ${ }^{6}$ and a histological study. ${ }^{16}$ Furthermore, basic research also showed that latanoprost, travoprost, tafluprost, and bimatoprost all have high affinity to prostaglandin F2 alpha (FP) receptor, and inhibit differentiation of adipocyte precursors through stimulating FP receptors. ${ }^{17,18}$ However, despite the reported data that travoprost has higher FP receptor affinity than bimatoprost, ${ }^{19}$ the incidence of DUES associated with travoprost does not appear to be higher than that of bimatoprost. Moreover, while tafluprost is reported to have higher affinity to the FP receptor than latanoprost,${ }^{20}$ the incidence 

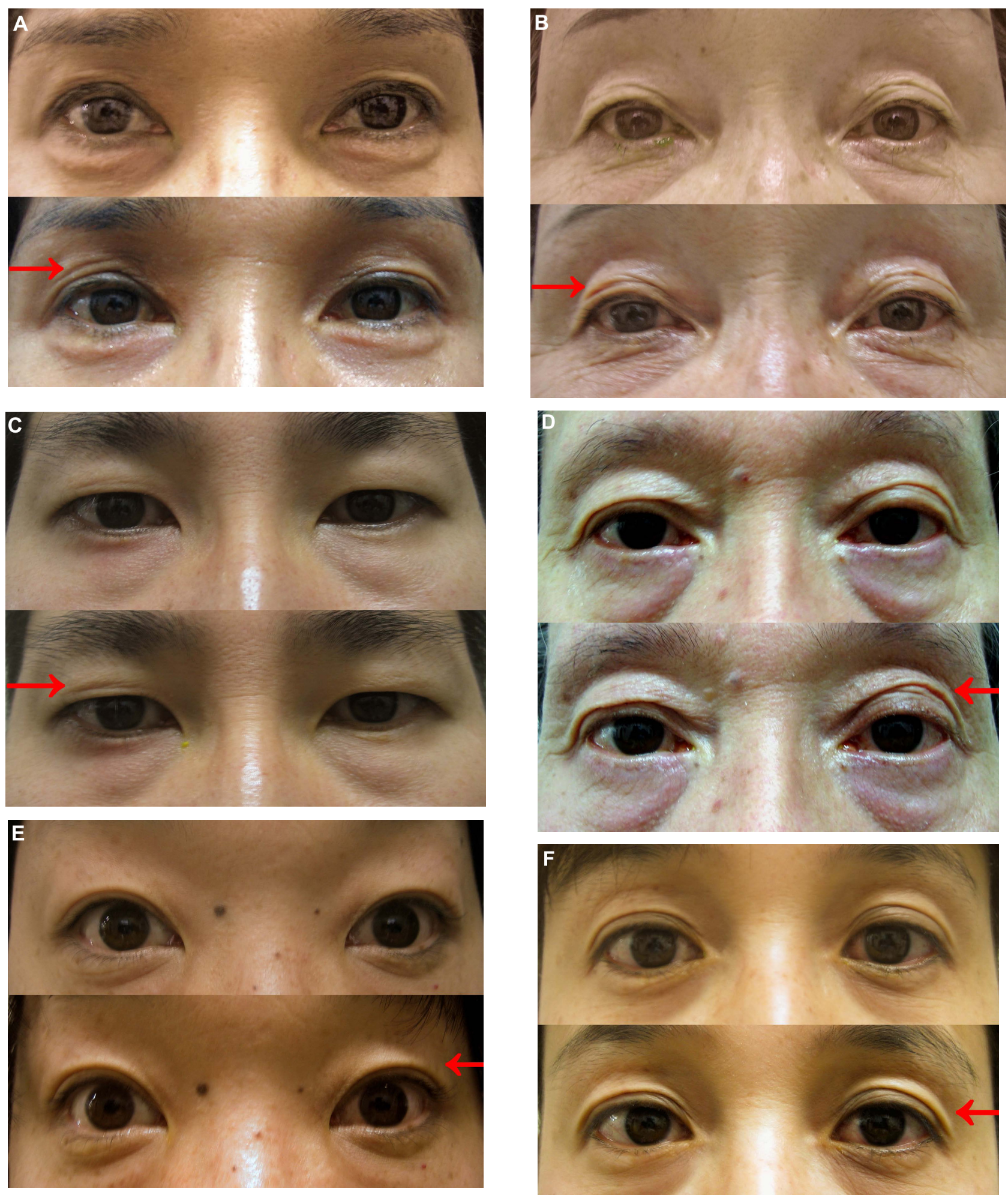

Figure 2 All six patients diagnosed with DUES after starting treatment with tafluprost in the unilateral eye. Photographs were taken before treatment (upper panels) and at the time of onset of DUES after starting treatment (lower panels).

Notes: (A) A 50-year old woman developed a new eyelid fold in the right eye (arrow) at 60 days after starting tafluprost treatment of the right eye. (B) A 61 -year old woman developed deepening of the eyelid fold (arrow) in the right eye at 60 days after starting tafluprost treatment of the right eye. (C) A 36-year-old man showed deepening of the eyelid fold (arrow) in the right eye at 90 days after starting tafluprost treatment of the right eye. He noticed DUES subjectively at 90 days of treatment. (D) A 66-year old man developed deepening of the eyelid fold (arrow) in the left eye at 60 days after starting tafluprost treatment of the left eye. (E) A 43-year old woman showed increased hollowing of the eyelid fold (arrow) in the left eye at 90 days after starting tafluprost treatment of the left eye. (F) A 45-year old woman developed deepening of the eyelid fold (arrow) in the left eye at 90 days after starting tafluprost treatment of the left eye.

Abbreviation: DUES, deepening of upper eyelid sulcus. 
Table 2 Comparison of factors affecting the incidence of DUES

\begin{tabular}{llll}
\hline & DUES & No DUES & $P$-value \\
\hline Number of patients & 6 & 26 & \\
Age (years) & $50.2 \pm 11.4$ & $53.0 \pm 15.2$ & $0.672^{\mathrm{a}}$ \\
Gender (male/female) & $2 / 4$ & $17 / 9$ & $0.194^{\mathrm{b}}$ \\
Refraction (diopter) & $-4.23 \pm 1.38$ & $-5.69 \pm 3.58$ & $0.338^{\mathrm{a}}$ \\
Baseline IOP $(\mathrm{mmHg})$ & $16.1 \pm 5.5$ & $15.6 \pm 2.0$ & $0.699^{\mathrm{a}}$ \\
Post-treatment IOP $(\mathrm{mmHg})$ & $13.8 \pm 5.5$ & $12.9 \pm 1.7$ & $0.449^{\mathrm{a}}$ \\
IOP reduction $(\mathrm{mmHg})$ & $2.3 \pm 1.1$ & $2.7 \pm 1.6$ & $0.525^{\mathrm{a}}$ \\
\hline
\end{tabular}

Notes: Values are mean \pm standard deviation; a'Student's $t$-test; 'Fisher's exact test Post-treatment IOP is the average of three measurements value after treatment. Abbreviations: DUES, deepening of upper eyelid sulcus; IOP, intraocular pressure.

of DUES obtained from the present study is lower than that of bimatoprost which has similar FP receptor affinity as latanoprost. In vitro studies that examined the effects of latanoprost, travoprost, bimatoprost, and tafluprost on preadipocyte differentiation reported that bimatoprost has the greatest anti-adipogenic effect, followed by travoprost and tafluprost with similar effects. ${ }^{21}$ These results are not in total agreement with the incidence of DUES caused by prostaglandin analogs. These observations suggest that the incidence of DUES is related to multiple mechanisms. Further studies are required to investigate the difference in DUES incidence of various prostaglandin analogs.

When DUES was evaluated by comparing eyelid photographs before and after administration of the medication, the rate of subjective awareness of DUES among objectively evaluated DUES-positive cases was $53 \%$ in bimatoprost. ${ }^{8}$ On the other hand, the present study showed a low subjective DUES awareness rate of $17 \%$ in tafluprost-treated patients. This result may suggest that tafluprost causes DUES not only at a lower incidence than bimatoprost, but also at a less degree of severity, resulting in a low rate of subjective awareness.

In the present study, no background factor related to the onset of DUES was identified. On the contrary, a study of bimatoprost ${ }^{8}$ reported that the DUES positive patients were older than DUES negative patients. Although the reason for the difference in results between studies is unclear, the small number of DUES positive patients compared to DUES negative patients in the present study may be a contributing factor.

There are some limitations in this study. First, the study is uncontrolled and conducted on a small number of patients from a single center. Second, DUES was diagnosed by assessing serial photographs, which is a qualitative method, because there is no established quantitative method for assessing DUES. Measuring the change in upper eyelid fat volume using
MRI may be a precise method, but repeated MRI examinations for assessing an adverse reaction of an anti-glaucoma agent cannot be justified. Under these circumstances, we attempted to standardize our method and assess as objectively as possible. Development of a quantitative and clinically applicable method for diagnosing DUES will be useful for future studies. In addition, although DUES signs were assessed by comparing the photographs before and after treatment and between the administered and non-administered eye in this study, it cannot be denied that physiological changes affected eyelid appearance. Third, the follow-up period was only 90 days, and the long-term DUES rate is not known. Despite these limitations, this study is the first prospective study that evaluates serial photographs comparing the administered eye and non-administered eye before and after treatment. Therefore, the results of this study are expected to have great impact on the choice of medication and consideration of side effects in glaucoma pharmacotherapy.

The results of the present study revealed that the prevalence of DUES caused by topical instillation of tafluprost was $19 \%$ after 90 days of treatment. Furthermore, the incidence of DUES was not related to the patient's background. Esthetic change during treatment may lead to diminished patients' trust and lowered adherence to treatment, eventually affecting the efficacy of glaucoma therapy. Further studies that investigate the prevalence of DUES associated with other prostaglandin analogs, compare the incidence between drugs or different upper eyelid morphologies, and examine the incidence in long-term treatment are required to accumulate evidence for educating patients on DUES as a side effect of prostaglandin analogs.

\section{Disclosure}

The authors report no conflicts of interest in this work.

\section{References}

1. Holló G. The side effects of the prostaglandin analogues. Expert Opin Drug Saf. 2007;6(1):45-52.

2. Peplinski LS, Albiani Smith K. Deepening of lid sulcus from topical bimatoprost therapy. Optom Vis Sci. 2004;81(8):574-577.

3. Filippopoulos T, Paula JS, Torun N, Hatton MP, Pasquale LR, Grosskreutz CL. Periorbital changes associated with topical bimatoprost. Ophthal Plast Reconstr Surg. 2008;24(4):302-307.

4. Tappeiner C, Perren B, Iliev ME, Frueh BE, Goldblum D. [Orbital fat atrophy in glaucoma patients treated with topical bimatoprost - can bimatoprost cause enophthalmos?] Klin Monbl Augenheilkd. 2008;225(5) 443-445. German.

5. Yam JC, Yuen NS, Chan CW. Bilateral deepening of upper lid sulcus from topical bimatoprost therapy. J Ocul Pharmacol Ther. 2009;25(5): $471-472$.

6. Jayaprakasam A, Ghazi-Nouri S. Periorbital fat atrophy - an unfamiliar side effect of prostaglandin analogues. Orbit. 2010;29(6):357-359. 
7. Nakakura S, Tabuchi H, Kiuchi Y. Latanoprost therapy after sunken eyes caused by travoprost or bimatoprost. Optom Vis Sci. 2011;88(9):1140-1144.

8. Aihara M, Shirato S, Sakata R. Incidence of deepening of the upper eyelid sulcus after switching from latanoprost to bimatoprost. Jpn J Ophthalmol. 2011;55(6):600-604.

9. Inoue K, Shiokawa M, Wakakura M, Tomita G. Deepening of the Upper Eyelid Sulcus Caused by 5 Types of Prostaglandin Analogs. J Glaucoma. Epub August 29, 2012.

10. Suh MH, Park KH, Kim DM. Effect of travoprost on intraocular pressure during 12 months of treatment for normal-tension glaucoma. Jpn J Ophthalmol. 2009;53(1):18-23.

11. Yang HK, Park KH, Kim TW, Kim DM. Deepening of eyelid superior sulcus during topical travoprost treatment. Jpn J Ophthalmol. 2009;53(2):176-179.

12. Maruyama K, Shirato S, Tsuchisaka A. Incidence of deepening of the upper eyelid sulcus after topical use of travoprost ophthalmic solution in Japanese. J Glaucoma. Epub August 23, 2012.

13. Ung T, Currie ZI. Periocular changes following long-term administration of latanoprost $0.005 \%$. Ophthal Plast Reconstr Surg. 2012;28(2): e42-e44.

14. Uusitalo H, Pillunat LE, Ropo A; Phase III Study Investigators. Efficacy and safety of tafluprost $0.0015 \%$ versus latanoprost $0.005 \%$ eye drops in open-angle glaucoma and ocular hypertension: 24-month results of a randomized, double-masked phase III study. Acta Ophthalmol. 2010;88(1):12-19.
15. Traverso CE, Ropo A, Papadia M, Uusitalo H. A phase II study on the duration and stability of the intraocular pressure-lowering effect and tolerability of Tafluprost compared with latanoprost. J Ocul Pharmacol Ther. 2010;26(1):97-104.

16. Park J, Cho HK, Moon JI. Changes to upper eyelid orbital fat from use of topical bimatoprost, travoprost, and latanoprost. Jpn J Ophthalmol. 2011;55(1):22-27.

17. Serrero G, Lepak NM. Prostaglandin F2alpha receptor (FP receptor) agonists are potent adipose differentiation inhibitors for primary culture of adipocyte precursors in defined medium. Biochem Biophys Res Commun. 1997;233(1):200-202.

18. Liu L, Clipstone NA. Prostaglandin F2alpha inhibits adipocyte differentiation via a $\mathrm{G}$ alpha q-calcium-calcineurin-dependent signaling pathway. J Cell Biochem. 2007;100(1):161-173.

19. Sharif NA, Kelly CR, Crider JY, Williams GW, Xu SX. Ocular hypotensive FP prostaglandin (PG) analogs: PG receptor subtype binding affinities and selectivities, and agonist potencies at FP and other PG receptors in cultured cells. J Ocul Pharmacol Ther. 2003;19(6):501-515.

20. Takagi Y, Nakajima T, Shimazaki A, et al. Pharmacological characteristics of AFP-168 (tafluprost), a new prostanoid FP receptor agonist, as an ocular hypotensive drug. Exp Eye Res. 2004;78(4): 767-776.

21. Choi HY, Lee JE, Lee JW, Park HJ, Lee JE, Jung JH. In vitro study of antiadipogenic profile of latanoprost, travoprost, bimatoprost, and tafluprost in human orbital preadiopocytes. J Ocul Pharmacol Ther. 2012;28(2):146-152.

\section{Clinical Ophthalmology}

\section{Publish your work in this journal}

Clinical Ophthalmology is an international, peer-reviewed journal covering all subspecialties within ophthalmology. Key topics include: Optometry; Visual science; Pharmacology and drug therapy in eye diseases; Basic Sciences; Primary and Secondary eye care; Patient Safety and Quality of Care Improvements. This journal is indexed on Submit your manuscript here: http://www.dovepress.com/clinical-ophthalmology-journal

\section{Dovepress}

PubMed Central and CAS, and is the official journal of The Society of Clinical Ophthalmology (SCO). The manuscript management system is completely online and includes a very quick and fair peer-review system, which is all easy to use. Visit http://www.dovepress.com/ testimonials.php to read real quotes from published authors. 